\title{
Spectral analysis of the gamma-ray background near the dwarf Milky Way satellite Segue 1: Improved limits on the cross section of neutralino dark matter annihilation
}

\author{
A. N. Baushev, S. Federici, and M. Pohl \\ DESY, 15738 Zeuthen, Germany and Institut für Physik und Astronomie, Universität Potsdam, 14476 Potsdam-Golm, Germany
}

(Received 16 May 2012; published 20 September 2012)

\begin{abstract}
The indirect detection of dark matter requires that dark matter annihilation products be discriminated from conventional astrophysical backgrounds. Here, we re-analyze GeV-band gamma-ray observations of the prominent Milky Way dwarf satellite galaxy Segue 1, for which the expected astrophysical background is minimal. We explicitly account for the angular extent of the conservatively expected gamma-ray signal and keep the uncertainty in the dark-matter profile external to the likelihood analysis of the gamma-ray data.
\end{abstract}

DOI: 10.1103/PhysRevD.86.063521

PACS numbers: 95.35.+d, 95.85.Pw, 95.30.Cq

\section{INTRODUCTION}

The physical nature of dark matter is still mysterious. One of the most probable hypotheses is that it is a mixture of equal quantities of weakly-interacting massive particles (WIMPs) and their antiparticles, generated in the early Universe. The detection of their annihilation products could be an attractive possibility to clarify the nature of these particles.

Dwarf satellite galaxies of the Milky Way are a class of objects that are very promising for detecting dark-matter annihilation. Though the dark-matter density in these objects is significantly lower than that at the Galactic Center, they typically contain no sources of cosmic rays, and therefore almost any high-energy signal detected from the dwarf galaxies could be the signal of dark-matter annihilation.

Tens of Milky-Way satellites are known to date. In our work we examine the dwarf galaxy Segue 1, since it combines several advantages: it is the closest satellite to Earth, it is located at high Galactic latitude (and, consequently, low gamma-ray background), and among the dwarf galaxies; Segue 1 is expected to produce the strongest dark-matter signal [1-3]. Because of it's proximity to the Sagittarius stream, the nature of Segue 1 has been disputed: it was argued to be a disrupted star cluster originally associated with the Sagittarius dSph [4]. However, a kinematic study of a larger member-star sample (66 stars compared to the previous 24-star sample) has recently confirmed that Segue 1 is indeed a dwarf satellite galaxy [3]. Table 1 in Ref. [5] shows that the line-of-sight annihilation integral through the dark-matter distribution of Segue 1 integrated over the angular size of the source is the largest of all Milky Way dwarf satellites.

Other approaches used in the literature include performing a joint likelihood analysis of several dwarf galaxies [5] and a Bayesian technique [6]. We do not find a joint analysis advantageous: actually, only a few objects can produce a flux comparable to Segue 1. Therefore, the statistics can only be improved marginally, while the merg- ing of several sources with various properties, densityprofile measurement errors, and backgrounds strongly increases the ambiguities of the analysis.

Our consideration is based on the assumption that the WIMP in question is the lightest supersymmetry (SUSY) particle, a neutralino. However, the only property of neutralinos we use is that they annihilate mainly into a pair of heavy particles (heavy quarks, gauge bosons, tau leptons etc.). So our conclusions are valid for any WIMPs for which the supposition applies.

We use data from the Fermi Large Area Telescope (LAT), since this telescope is currently the most sensitive to the photons that can be generated by the annihilation of neutralinos with mass roughly between $10 \mathrm{GeV}$ and $1 \mathrm{TeV}$. Currently operating imaging Cherenkov telescopes are primarily sensitive to gamma rays with energies $\gtrsim 200 \mathrm{GeV}$ and hence constrain very massive dark-matter particles [7]. Fermi-LAT data have already been used in several articles [2,5,8,9]. However, all of them, except for Ref. [8], considered Segue 1 as a point source. We perform an accurate spectral analysis and explicitly account for the spatial extent of the source, using more data than [8], which allows us to improve the result. In Ref. [5], the Segue 1 darkmatter distribution is modeled with a Navarro-Frenk-White profile [10], a centrally concentrated density distribution, thus leading to an enhanced gamma-ray signal. We use a template for the angular distribution based on the significantly more conservative Einasto profile with arbitrary scale factor, diffuse and point-source backgrounds. The Einasto profile predicts a spatially more extended signal, and the nominal annihilation flux is weaker than in Ref. [5]. As we will show in Sec. V, the Einasto model predicts an annihilation signal that is only marginally higher than the lowest limit for any density distribution compatible with the stellar dynamics of Segue 1. We further investigate a possible position-dependent Sommerfeld enhancement [11] of the annihilation rate. As both the total annihilation signal and the source extent are affected by the choice of dark-matter profile and 
enhancement factors, the uncertainties project onto the gamma-ray-based upper limits in a nontrivial way.

\section{MODEL DESCRIPTION}

In order to avoid ambiguity, we hereafter suppose that the dark-matter particle is identical to the antiparticle. If the WIMP is a Dirac particle (which is not the case for neutralinos), then the annihilation rate halves and all limits on the cross sections would be weaker by a factor 2 . On the other hand, the main purpose of our work is to compare the limits obtained with the cross section estimate from darkmatter abundance $\langle\sigma v\rangle_{a} \simeq 3 \times 10^{-26} \mathrm{~cm}^{3} \mathrm{~s}^{-1}$ [12] (see below). This value is also increased by the same factor 2 , if the dark-matter particle is not a Majorana particle. Therefore, the ratio between the limit obtained and $\langle\sigma v\rangle_{a}$ is not sensitive to the nature of the dark matter in this sense.

The annihilation signal (i.e., the number of photons $d \Re$ arriving at the local observer from a solid angle element $d \Omega$, per unit time interval $d t$, per an area $d A$, and an energy interval $d E$ ) in the general case is

$$
I=\frac{d \Re}{d A d \Omega d E d t}=\frac{\langle\sigma v\rangle_{c}}{8 \pi m_{\chi}^{2}} \cdot F \cdot \frac{d N}{d E},
$$

where $d N / d E$ is the spectrum of the photons produced by a single annihilation event, and

$$
F\left(r_{p}\right)=\int_{\mathrm{LOS}} C \frac{\langle\sigma v\rangle}{\langle\sigma v\rangle_{c}} \rho^{2} d l .
$$

$F\left(r_{p}\right)$ is the line-of-sight integral of the multiplication of tree factors through the density profile of the source at the projected radius $r_{p} . C=\left\langle\rho^{2}\right\rangle /\langle\rho\rangle^{2}$ is the so-called boost factor, which takes into account the enhancement of the annihilation signal by dark-matter substructures that may be present. The question of possible substructures in Segue 1 is extremely vague, and we will not consider it, accepting $C \equiv 1$. The factor $\langle\sigma v\rangle /\langle\sigma v\rangle_{c}$ allows for a possible dependence of $\langle\sigma v\rangle$ on particle speed, e.g., the Sommerfeld effect. $\langle\sigma v\rangle_{c}$ is a characteristic value chosen such that for absent velocity dependence $\langle\sigma v\rangle=\langle\sigma v\rangle_{c}=$ constant.

With knowledge of the distance, $F\left(r_{p}\right)$ fully describes the angular distribution. We should mention that quantities such as $J(\Omega) \equiv L(\Omega)=\int_{\Omega(r)} F\left(r_{p}[\Omega /]\right) d \Omega /$, where $J$ or $L$ are considered as a function of the angle, are of more common use in the literature. Being defined so, they depend on the distance to the source, contrary to $F\left(r_{p}\right)$. We set the distance to Segue 1 to $23 \mathrm{kpc}$.

The mass of Segue 1 within a certain radius is measured by observations of a few $(<100)$ member stars, but the density profile is assumed (e.g., Refs. $[1,13])$. To describe the signal profile we used four models. The first two assume that $\langle\sigma v\rangle$ does not depend on the velocity.

The first model (hereafter the standard model) is based on the Einasto density profile

$$
\rho=\rho_{s} \exp \left[-2 n\left\{\left(\frac{r}{r_{s}}\right)^{1 / n}-1\right\}\right]
$$

with the parameters of Eq. (3) in accordance with Ref. [14] as $\rho_{s}=1.1 \times 10^{8} M_{\odot} \mathrm{kpc}^{-3}, r_{s}=0.15 \mathrm{kpc}$, and $n=3.3$. Further we assume for the standard profile $\langle\sigma v\rangle=\langle\sigma v\rangle_{c}$. It is worth mentioning that the enclosed mass and the function $F\left(r_{p}\right)$ can be experimentally defined much better than the individual parameters $\rho_{s}, r_{s}$, and $n$. Some authors [8] use a completely different set of parameters, which corresponds to a quite similar $F\left(r_{p}\right)$. We note that the total integral $J_{\text {tot }}=\oint F d \Omega=10^{19.2} \mathrm{GeV}^{2} \mathrm{srcm}^{-5}$ in our case (and cgs units) is smaller than the $J_{\text {tot }}=$ $10^{19.6} \mathrm{GeV}^{2} \mathrm{sr} \mathrm{cm}^{-5}$ used in Ref. [5].

We should emphasize significant uncertainties of the Segue 1 density profile: almost all the stars lie inside $10^{\prime}$ $(\simeq 67 \mathrm{pc})$ from the center [3], which is significantly smaller than $r_{s}$. In order to test how sensitive the annihilation signal is to the choice of profile, we used a second (minimal) model. The assumptions of the minimal model are that the density distribution beyond a radius of $67 \mathrm{pc}$ and the total mass inside this radius coincide with those of the standard model (3), but the dark matter is homogeneously distributed in the inner $67 \mathrm{pc}$. In this case the predicted signal is minimal among the profiles compatible with observations. This conservative choice of density profile also renders the angular extent of the annihilation signal larger than in the case of a more spiked density profile, for which a point-source analysis may be suitable.

Among all the scenarios with $\langle\sigma v\rangle$ depending on $v$, we consider the Sommerfeld effect [11]. If the dark-matter particles interact only via standard gauge bosons, the effect arises only for heavy WIMPs, with masses in the $\mathrm{TeV}$ range, but if there are lighter mediator particles, the enhancement appears even in the case of low-mass WIMPs [15]. A similar explanation has been invoked for the PAMELA positron excess [16]; however, any discussion of the PAMELA results or of the boosting necessary for the dark-matter interpretation of the PAMELA excess goes far beyond the scope of this article. We would like to emphasize only two essential points. First, the Sommerfeld effect can take place only if there is some new relatively light mediator field for the majority of the WIMP masses under consideration in this article (except for the highest ones). Second, if the Sommerfeld effect takes place, $\langle\sigma v\rangle$ depends on the collision velocity, and we should indicate the characteristic velocity when we obtain experimental constrains of the cross section.

To account for the Sommerfeld effect we used two models. Both of them are based on the Einasto density profile, as the standard model. This allows us to find $\phi(r)$, the gravitational potential of Segue 1. Roughly speaking, if the Sommerfeld effect occurs, then $\langle\sigma v\rangle \sim 1 / v$. The question is how $v$ depends on radius, $r$. We scale $\langle\sigma v\rangle$ using as characteristic velocity the escape velocity from the center of Segue $1, v_{c}=2 \sqrt{-\phi(0)} \simeq 60 \mathrm{~km} \mathrm{~s}^{-1}$, 


$$
\langle\sigma v\rangle=\frac{v_{c}}{v}\langle\sigma v\rangle_{c} .
$$

For both the models taking into account the Sommerfeld effect the limits on the cross section we obtain are considered to mean the constraints on the quantity $\langle\sigma v\rangle_{c}$, the $\langle\sigma v\rangle$ at the average collision velocity $v_{c} .\langle\sigma v\rangle$ at the decoupling epoch could be much lower in this case.

Besides the absolute intensity, the Sommerfeld effect affects the angular profile: we should take into account the velocity distribution of the particles as a function of $r$. The velocity distribution properties are still not quite clear [17]. We consider two possible hypotheses about the distribution of average collision speed $v$ inside Segue 1. We may suppose that the speed is proportional to the escape velocity, or, for simplicity, $v=2 v_{\text {esc }} / \sqrt{2}=2 \sqrt{-\phi(r)}$. A very similar situation takes place if radial motion of the particles dominates [18]. Hereafter we call this model virial.

In the opposite case, when the distribution function is supposed to be more or less isotropic, it is reasonable to suppose $v=\sqrt{2} v_{\text {orb }}(r)$, where $v_{\text {orb }}(r)$ is the orbital speed at radius $r$. This situation resembles the well-known isothermal dark-matter halo and should be typical for the halo center [19]. Moreover, the experimentally observed [3] small velocity dispersion of stars at the center of Segue 1, $\sim 3.7 \mathrm{~km} \mathrm{~s}^{-1}$, favors this scenario. We name this model orbital.

The signal profile as described by each of the four models is shown in Fig. 1.

The error in the experimental determination of the multiplier $F\left(r_{p}\right)$ slightly depends on $r$ and can be estimated [14] as

$$
\sigma\left(\log _{10} F\right) \simeq 0.6
$$

If we assume the uncertainty in $\log _{10} F$ to be Gaussian, then the 2- $\sigma$ confidence interval of $F\left(r_{p}\right)$ corresponds to an

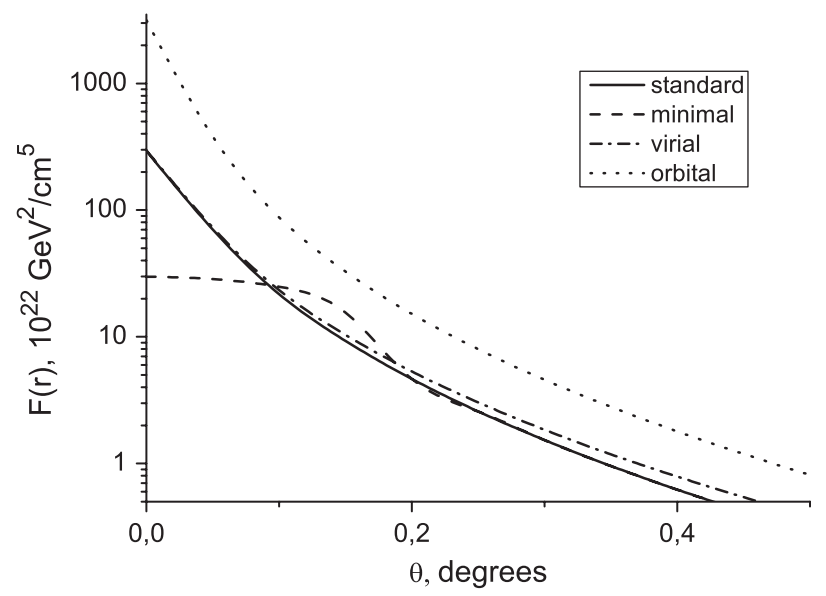

FIG. 1. The angular distribution of the signal for the four profiles used in this article. Here $\theta$ is the angular distance from the center of the source, $F(r)$ is defined by (2) using $C=1$ and $\langle\sigma v\rangle=\langle\sigma v\rangle_{c}$. additional multiplier in Eq. (1) extending over the range $0.063-16$. It is obvious that this is the main source of uncertainty in the determination of the upper limit on the cross section. However, $F\left(r_{p}\right)$ is an external factor in the likelihood analysis of the Fermi-LAT data, provided the angular profile is realistic, and therefore for a given darkmatter profile and signal boosting the limits on $\langle\sigma v\rangle$ will to first order scale with the error factor in $F\left(r_{p}\right)$.

The angular size of the source is comparable to the angular resolution of Fermi-LAT above $10 \mathrm{GeV}$; for the standard model $68 \%$ of the signal is expected within $0.17^{\circ}$ and $95 \%$ of the signal within $0.54^{\circ}$ of the source center. As we used a binned likelihood analysis, we wanted to avoid a dependence of the model on the position in the map grid. For that purpose we smoothed the profiles: instead of $F\left(r_{p}\right)$ defined by (2) we used $\tilde{F}\left(r_{p}\right) \equiv \int_{\Delta \Omega} F\left(r_{p}\right) d \Omega / \Delta \Omega$, where $\Delta \Omega$ is $\sim 0.037^{\circ} \times 0.037^{\circ}$, which is slightly smaller than the pixel size and corresponds to an area $15 \mathrm{pc} \times 15 \mathrm{pc}$ at the source. The smoothing kernel is about a factor 2.5 narrower than the angular resolution of Fermi-LAT above $10 \mathrm{GeV}$ and its particular choice has no impact on the results of the likelihood fit.

The question of the photon spectrum produced by neutralino annihilation is, generally speaking, quite complex. Annihilation into a pair of particles (quarks, leptons, gauge bosons etc.) is the most likely process. The channels with light final states are suppressed by helicity, and the primary products of the process are heavy particles ( $b, t, c$-quarks, $W, Z$-bosons, $\tau$ leptons). Let us denote the branching ratio of each individual channel by $k$ (for instance, $k_{\tau \tau}$ is the average fraction of annihilations resulting in a $\tau \bar{\tau}$ pair generation). The heavy primary particles are unstable and decay into stable products including photons (mainly from pion decays). We calculated the spectrum of photons generated by various annihilation channels with the DARKSUSY package [20].

The real photon spectrum is a linear combination of the spectra corresponding to separate channels with coefficients $k_{i}$. Channel contributions $k_{i}$ (and, consequently, the spectrum) depend on the choice of SUSY model. To avoid a complete scan of the SUSY parameter space, we use the spectra of separate channels to obtain upper limits on the products $k_{i} \sigma$. For each neutralino mass we use two types of spectral models: the spectra corresponding to pure annihilation into $b \bar{b}$ and $\tau \bar{\tau}$, respectively. We reproduce in Fig. 2 the photon spectra generated by the annihilation of a pair of $200-\mathrm{GeV}$ neutralinos through various channels (the spectra are normalized as if the channel under consideration was the only channel of annihilation). As we can see, the spectra of all channels, except for $\tau \bar{\tau}$ and $t \bar{t}$, are very similar to that of the $b \bar{b}$ channel. Therefore, by placing a limit on $k_{b b} \sigma$, we actually provide a very good approximation of the upper bound on $\left(k_{b b}+k_{c c}+k_{W W}+k_{Z Z}\right) \sigma$.

Note that, for a significant part of SUSY parameter space, $\left(k_{b b}+k_{c c}+k_{W W}+k_{Z Z}\right)$ is close to 1 . The relative 


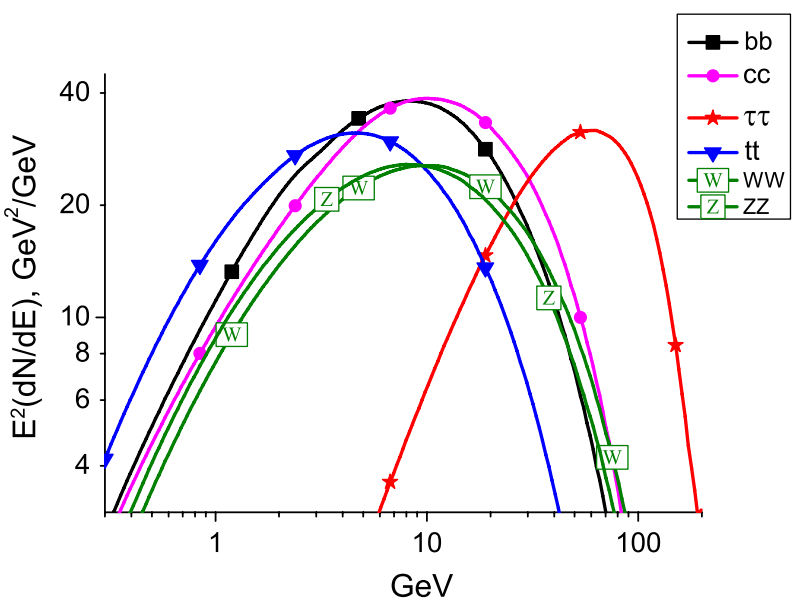

FIG. 2 (color online). The photon spectra generated by the annihilation of a pair of $200-\mathrm{GeV}$ neutralinos through various channels (the spectra are normalized as if the channel under consideration were the only channel of annihilation). The $b \bar{b}, c \bar{c}$, $\tau \bar{\tau}, t \bar{t}, W \bar{W}$, and $Z \bar{Z}$ are represented by the lines with squares, circles, stars, triangles, $W$, and $Z$, respectively.

width of the $\tau \bar{\tau}$ channel is usually significantly smaller than 1 , but the spectrum is very hard and, consequently, easily distinguished, since the background rapidly decreases with energy. The $t \bar{t}$ spectrum is somewhat softer than that of $b \bar{b}$ decay, but we choose not to consider it as the third spectral model for a number of reasons. First of all, this channel is closed for light neutralinos because of the large mass of the $t$-quark. Second, $\left(\left(k_{b b}+k_{c c}+\right.\right.$ $\left.k_{W W}+k_{Z Z}\right)$ ) is typically at least comparable with $k_{t t}$ [21]. Third, the spectrum of the $t \bar{t}$ channel does not differ very much from that of $b \bar{b}$.

We consider an array of neutralino masses that covers the expected mass range [22] approximately uniformly in $\log E$ : 40, 50, 63, 80, 100, 126, 159, 199, 251, 316, 398, 501, 631, 794, 1000, 1259, 1585, 1995, 2512, 3162, 3981, and $5011 \mathrm{GeV}$. In total we use 44 spectral models: a $b \bar{b}$ and a $\tau \bar{\tau}$ model for each of 22 neutralino masses. The spatial models are, of course, independent on the neutralino mass.

\section{OBSERVATIONS OF SEGUE 1 WITH FERMI-LAT}

We searched for evidence of gamma-ray emission from the dwarf spheroidal galaxy Segue 1 using data from LAT, the principal instrument on board the Fermi spacecraft, a description of which is given in Ref. [23].

Data analysis was performed with the Fermi-LAT SCIENCETOOLS version 9.23.1, using the post-launch P7SOURCE_V6 instrument response functions. The cosmic-ray background was reduced by choosing the P7SOURCE class which only includes events with high probability of being photons. We also require a zenithangle cut of $100^{\circ}$ to minimize the spill-over of photons from the Earth limb, excluding time intervals where any part of the region of interest was beyond the zenith-angle limit. In addition, data were not taken while the observatory was transiting the South Atlantic Anomaly and excluded when the rocking angle exceeded $52^{\circ}$. The data used in this work came from observations in the period from August 8, 2008 to February 4, 2012.

A binned likelihood analysis is performed on a map with $0.05^{\circ}$ pixel size in gnomonic (TAN) projection, measuring $10^{\circ}$ on a side and centered on $\alpha_{J 2000}=151.767^{\circ}$ and $\delta_{J 2000}=16.0819^{\circ}$, the nominal position of Segue 1 . We only used photons with reconstructed energy greater than $2.4 \mathrm{GeV}$, for which the $68 \%$-containment radius of the point-spread function is narrower than $\sim 0.45^{\circ}$. The annihilation spectra are always substantially harder than those of the background, and therefore a high-energy threshold can help to minimize background contamination.

The resulting background model includes seven sources listed in the second LAT source catalog [24], and the LAT standard Galactic diffuse emission component gal_2yearp7v6_v0.fits along with the corresponding isotropic template iso $\_p 7 v 6$ source.txt that accounts for extragalactic emission and residual cosmic-ray contamination [25]. All seven sources lie outside our region of interest and are modeled as point sources with power-law energy spectra. Their spatial and spectral parameters were kept fixed at the values given in the catalog, while we permitted the normalizations of the diffuse components to freely vary.

Segue 1 is characterized by a spatial template of $2^{\circ}$ diameter with a resolution of $0.05^{\circ}$, following Eqs. (2) and (3). Despite of the fact that the spatial binning is slightly larger than the smoothing kernel applied to the Segue 1 profile, the results of the likelihood analysis are not affected. Analyses with different binning scales show, in fact, changes in the integral flux upper limits less than $1 \%$.

As described above, we use two different model spectra for the self-annihilation of neutralinos into quarks $(b \bar{b})$ and leptons $(\tau \bar{\tau})$ for each of the four model profiles (standard, minimal, virial, orbital). We tested 22 values of dark-matter particle mass in the range from $40 \mathrm{GeV}$ to $5011 \mathrm{GeV}$.

\section{ANALYSIS AND RESULTS}

We analyzed data between $2.4 \mathrm{GeV}$ and $300 \mathrm{GeV}$. The low-energy limit was a priori chosen to optimize the constraints on the neutralino masses above $100 \mathrm{GeV}$. The normalization of the spatial template of Segue 1 is the relevant free parameter since we keep the position fixed. No significant gamma-ray signal was detected. We thus derive integral-flux upper limits over the energy range $2.4 \mathrm{GeV}-300 \mathrm{GeV}$ using the profile likelihood technique. Table I provides $95 \%$ confidence-level upper limits for dark-matter self-annihilation emission from Segue 1 (standard model only). 
TABLE I. Integral-flux upper limits above an energy threshold of $2.4 \mathrm{GeV}$ for different WIMP masses (standard model). The confidence level is $95 \%$ and the units $10^{-11} \mathrm{ph} \mathrm{cm}^{-2} \mathrm{~s}^{-1}$.

\begin{tabular}{lcc}
\hline \hline$m_{\chi}, \mathrm{GeV}$ & Flux upper limit, $b \bar{b}$ & Flux upper limit, $\tau \bar{\tau}$ \\
\hline 50 & 7.98 & 7.65 \\
100 & 8.43 & 5.83 \\
501 & 7.42 & 3.44 \\
1000 & 6.64 & 2.99 \\
5011 & 5.24 & 2.80 \\
\hline \hline
\end{tabular}

Figure 3 shows the upper limits on the integrated flux as a function of the neutralino mass for the two annihilation channels for the four models. We note that the $b \bar{b}$ final state predicts on average a smaller photon energy than the $\tau \bar{\tau}$ final state (cf. Fig. 2). Therefore, the background contamination is more serious and, consequently, the flux upper limits are lower for the $\tau \bar{\tau}$ channel.

To be noted from Fig. 1 is the effect of the shape of the annihilation profile, $F\left(r_{p}\right)$. The $30 \%$ difference between the best and worst upper limit reflect the deterioration in sensitivity arising from source extent. The flux upper limits do not depend on the normalization of the annihilation profile, the astrophysical factor $J$.

The impact of using P7SOURCE class events, instead of the older P6_V3_DIFFUSE, was also investigated. Differences of the two data sets slightly affect the results of the upper limits on the velocity-averaged selfannihilation cross section of neutralinos. The analysis performed with the Pass- 6 data provides more constraining upper limits compared with the Pass-7 data, of around $10 \%$ for the low-mass neutralino (i.e., $\mathrm{m}_{\chi}<100 \mathrm{GeV}$ for $b \bar{b}$ and $\mathrm{m}_{\chi}<501 \mathrm{GeV}$ for $\tau \bar{\tau}$ model) and around $20 \%$ for those with higher mass. Note that this change is likely due

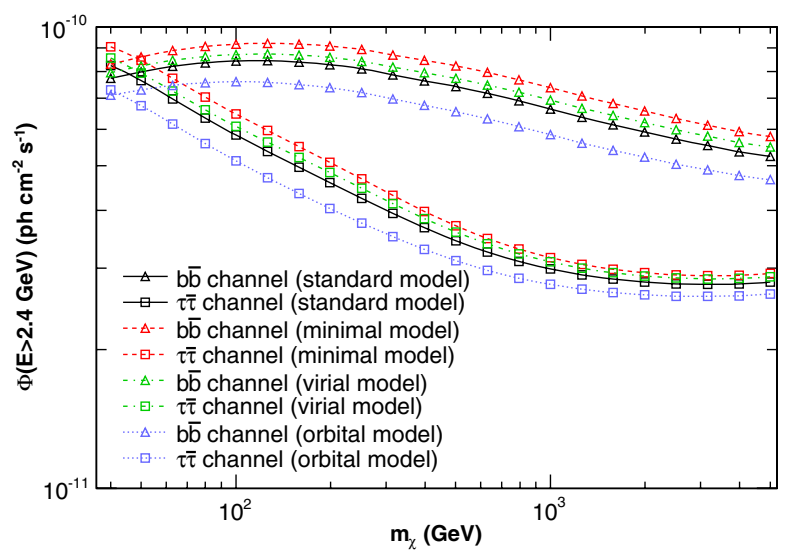

FIG. 3 (color online). 95\%-C.L. integral flux upper limits for Segue 1. The curves with the same color represent the two channels $100 \% b \bar{b}$ (triangular markers) and $100 \% \tau \bar{\tau}$ (square markers) for a specific model. All of the four models described in the text are shown. to a statistical fluctuation. The templates for diffuse and isotropic emission are different for Pass 6 and Pass 7, and they dominate the likelihood fit. In the Pass-7 list of events between $2.4 \mathrm{GeV}$ and $300 \mathrm{GeV}$ reconstructed energy, we find 7 events within $0.5^{\circ}$ of the nominal position of Segue 1 ( 5 events in the Pass- 6 event list). The entire region of interest comprises 920 events, or 9.2 per square-degree on average.

Comparing the standard profile with the point-source limit, we conclude that accounting for the source extent in the likelihood analysis increases the upper limits by typically $20 \%-25 \%$, but only $10 \%-15 \%$ for $\tau \bar{\tau}$ models with large particle mass, for which most of the gammarays have energies $\gtrsim 100 \mathrm{GeV}$.

\section{DISCUSSION}

Figure 4 represents the restrictions on $\langle\sigma v\rangle$ imposed by our analysis, assuming $k_{b b}=1$ or $k_{\tau \tau}=1$. Note that the restrictions do not take into account the uncertainty [Eq. (5)] in the dark-matter distribution. The systematic and statistical uncertainties arising from modeling the stellar kinematics of Segue 1 are in fact the dominant source of error, for which we do not know the probability distribution. In any case, it is not a purely statistical uncertainty.

We can draw several conclusions. First of all, the limits for the standard and minimal models differ only weakly, suggesting that the limits obtained using the Einasto profile are quite conservative and not very sensitive to the shape of the density profile. The limits for the standard and virial models almost coincide. This is a consequence of the fact that the central region of the source is rather small, and its angular size is smaller than $r_{s}$. As a result, $\phi$ (and so the average particle speed) changes only slightly inside the source.

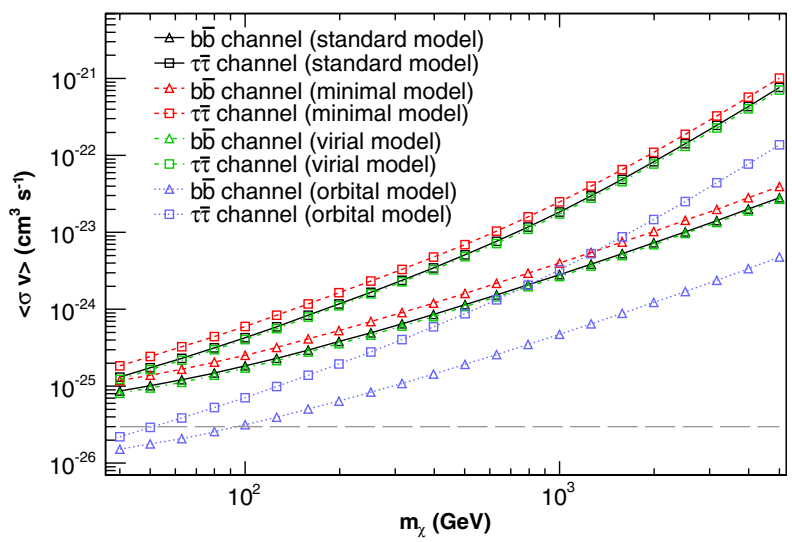

FIG. 4 (color online). 95\%-C.L. upper limits on $\langle\sigma v\rangle$ as a function of the neutralino mass for $k_{b b}=1$ (curves with triangular markers) and $k_{\tau \tau}=1$ (curves with square markers) for each of the four models. The horizontal dashed line shows the canonical value of the self-annihilation cross section for a thermal WIMP $\left(\langle\sigma v\rangle_{a} \simeq 3 \times 10^{-26} \mathrm{~cm}^{3} \mathrm{~s}^{-1}\right)$. 
The only model that excludes the relic abundance cross section $\langle\sigma v\rangle_{a} \simeq 3 \times 10^{-26} \mathrm{~cm}^{3} \mathrm{~s}^{-1}$ (in the area of small neutralino masses) is the orbital. This is due to the fact that $v_{\text {orb }} \rightarrow 0$ if the central density of the halo is finite. As a result, the signal in the center is significantly boosted. Unfortunately, the limitation is significant only for lowmass neutralinos and in the presence of the Sommerfeld enhancement. As we have already discussed the Sommerfeld effect for low-mass particles can appear only if the WIMPs interact via some low-mass mediator boson, discriminated from the known gauge bosons. So we can exclude only rather exotic dark-matter models.

A direct comparison of our results with those of Ref. [5] is difficult, because they chose to include in the likelihood analysis the substantial statistical uncertainty in the $J$-factor derived from fitting a specific dark-matter profile to the stellar-kinematic data. Our limit on $\langle\sigma v\rangle$ for the, e.g., $b \bar{b}$ channel with DM-mass $100 \mathrm{GeV}$ is a factor 5 better than that of Ref. [5], and it is marginally lower than those quoted ibidem for Draco, Ursa Major II, Ursa Minor, and Coma Berenices. As described in Sec. IV, using Pass-7 event selection and the extended source profile would increase the upper limit by $\sim 40 \%$, whereas the longer observing time should decrease it by a similar percentage, and thus the effects should roughly balance each other.

The marginal improvement in the upper limits presented here compared with those in Ref. [5] quoted for Ursa Major II and Coma Berenices is therefore very reasonable. However, our nominal $J$-factor for Segue 1 is a factor 2.5 lower than that used in Ref. [5], whereas their quoted uncertainty in $J$ is a factor 3.4 which they include in the likelihood analysis. Again, one might expect that accounting for the uncertainty in $J$ should roughly compensate the higher nominal $J$, but our results suggest that we would need to set $\log J=18.5$ to match their upper limit, a value that is $2 \sigma$ off their nominal $J$.

As we have already mentioned, the channels $(b \bar{b}+c \bar{c}+$ $W \bar{W}+Z \bar{Z}$ ) dominate for the majority of SUSY models, and the total spectrum differs little from that of $b \bar{b}$ annihilation. Therefore we may consider the limit given by the $b \bar{b}$ model to be with reasonable accuracy the limit on the total cross section.

But what constraints can the limits place on dark-matter models? First, all models for which the dark matter has never been in thermodynamic equilibrium should predict the annihilation cross section to be much lower than the limits shown in Fig. 4. If the dark matter was once in thermodynamic equilibrium, we can estimate the cross section at that epoch from the dark-matter abundance as $\langle\sigma v\rangle_{a} \simeq 3 \times 10^{-26} \mathrm{~cm}^{3} \mathrm{~s}^{-1}$ for Majorana particles [12]. As we can see, the upper limits never reach $\langle\sigma v\rangle_{a}$, except for the orbital model; for all other profiles they are at least a factor 4 higher.

On the other hand, the difference is not so large, and one might suppose that our results exclude large cross section enhancements, like strong Sommerfeld effect or high boost factor $C$. Unfortunately, even this conclusion is, generally speaking, questionable. When dark matter was formed, its average speed was tens of thousand of kilometers per second. Now it is of the order of hundreds of kilometers per second [18]. If the $s$-channel of annihilation dominates (which is typically the case), $\langle\sigma v\rangle \simeq$ const $+\alpha v^{2}$. The $p$-channel dominates more rarely, in which case $\langle\sigma\rangle \simeq$ const $+\alpha v^{2}$ [21]. We conclude that $\langle\sigma v\rangle$ for small velocities, i.e., for the present epoch, can be similar to its value at freeze-out (in the first case) or down to 100 times less (in the second case). In the latter instance, we cannot even exclude a factor of a hundred enhancement. We may only disfavor a very large boosting $(>500)$.

As we can see, the experimental results obtained do not significantly constrain dark-matter models (except for the quite weak limitation on the boost-factor or Sommerfeld enhancement). A rational question at this point is: What observations could improve the limit? First of all, a better measurement of the dark-matter profile of Segue 1 would reduce the systematic uncertainties [cf. Eq. (5)], but not necessarily the upper limits. However, even then the thermal freeze-out cross section $\langle\sigma v\rangle_{a}$ would not be challenged for any neutralino mass, whereas we should reach at least this value to place any reasonable constraints on dark-matter models. As we can see in Fig. 4, the limits on $\langle\sigma v\rangle$ must be improved by 2 orders of magnitude for particle masses around $1 \mathrm{TeV}$. At higher masses the current generation of Cherenkov telescopes is more sensitive than Fermi-LAT [7]. For satellite-based detectors of GeV-band gamma-rays this is hardly possible because one would need to operate in the background-dominated mode, for which the sensitivity increases only with the square root of the observing time. The isotropic, presumably extragalactic, background alone provides an integrated photon flux (above $2.4 \mathrm{GeV}$ ) per $0.2^{\circ}$-pixel that is $2.5 \%$ of our $b b$-model upper limit. Improvements to the point-spread function of the gamma-ray detector would not help on account of the intrinsic angular extent of the source. Observations of gamma-rays with energies $\sim 20 \mathrm{GeV}$ may still be conducted with moderate sky background, although not in the photon-counting limit. Therefore, large Cherenkov telescopes such as theCherenkov Telescope Array seem to be a promising alternative. The main advantage of these instruments is their huge effective area $\sim 10^{6} \mathrm{~m}^{2}[7,26]$ which could be enough to reach $\langle\sigma v\rangle_{a}$ even for heavy WIMPs. The only significant weakness of Cherenkov detectors is their relatively high energy threshold $(\sim 50 \mathrm{GeV})$, i.e., they are fundamentally unable to detect the annihilation of WIMPs of lower mass. On the other hand, for a large part of reasonable dark-matter models (like mSUGRA or CMSSM) a low-mass WIMP will very soon be excluded (or detected) by the LHC. 


\section{ACKNOWLEDGMENTS}

Financial support by Bundesministerium für Bildung und Forschung through DESY-PT, grant 05A11IPA, is gratefully acknowledged. BMBF assumes no responsibility for the contents of this publication. We acknowledge support by the Helmholtz Alliance for Astroparticle Physics HAP funded by the Initiative and Networking Fund of the Helmholtz Association. The Fermi LAT Collaboration acknowledges generous ongoing support from a number of agencies and institutes that have supported both the development and the operation of the LAT as well as scientific data analysis. These include the National Aeronautics and Space Administration and the Department of Energy in the United States, the Commissariat à l'Energie Atomique and the Centre
National de la Recherche Scientifique/Institut National de Physique Nucléaire et de Physique des Particules in France, the Agenzia Spaziale Italiana and the Istituto Nazionale di Fisica Nucleare in Italy, the Ministry of Education, Culture, Sports, Science and Technology (MEXT), High Energy Accelerator Research Organization (KEK) and Japan Aerospace Exploration Agency (JAXA) in Japan, and the K. A. Wallenberg Foundation, the Swedish Research Council and the Swedish National Space Board in Sweden. Additional support for science analysis during the operations phase is gratefully acknowledged from the Istituto Nazionale di Astrofisica in Italy and the Centre National d'Études Spatiales in France.
[1] G. D. Martinez, J. S. Bullock, M. Kaplinghat, L. E. Strigari, and R. Trotta, J. Cosmol. Astropart. Phys. 06 (2009) 014.

[2] R. Essig, N. Sehgal, L.E. Strigari, M. Geha, and J.D. Simon, Phys. Rev. D 82, 123503 (2010).

[3] J. D. Simon et al., Astrophys. J. 733, 46 (2011).

[4] M. Niederste-Ostholt, V. Belokurov, N. W. Evans, G. Gilmore, R. F. G. Wyse, and J.E. Norris, Mon. Not. R. Astron. Soc. 398, 1771 (2009).

[5] M. Ackermann et al. (Fermi-LAT Collaboration), Phys. Rev. Lett. 107, 241302 (2011).

[6] M.N. Mazziotta, F. Loparco, F. de Palma, and N. Giglietto, arXiv:1203.6731.

[7] A. Charbonnier et al., Mon. Not. R. Astron. Soc. 418, 1526 (2011).

[8] P. Scott, J. Conrad, J. Edsjö, L. Bergström, C. Farnier, and Y. Akrami, J. Cosmol. Astropart. Phys. 01 (2010) 031.

[9] A. Geringer-Sameth and S. M. Koushiappas, Phys. Rev. Lett. 107, 241303 (2011).

[10] J. F. Navarro, C. S. Frenk, and S. D. M. White, Astrophys. J. 490, 493 (1997).

[11] J. Hisano, S. Matsumoto, M. M. Nojiri, and O. Saito, Phys. Rev. D 71, 063528 (2005).

[12] G. Bertone, D. Hooper, and J. Silk, Phys. Rep. 405, 279 (2005).

[13] M. Geha, B. Willman, J. D. Simon, L. E. Strigari, E. N. Kirby, D. R. Law, and J. Strader, Astrophys. J. 692, 1464 (2009).
[14] J. Aleksić et al., J. Cosmol. Astropart. Phys. 06 (2011) 035.

[15] J. L. Feng, M. Kaplinghat, and H. B. Yu, Phys. Rev. D 82, 083525 (2010).

[16] O. Adriani et al., Nature (London) 458, 607 (2009).

[17] A. N. Baushev, Mon. Not. R. Astron. Soc. 420, 590 (2012).

[18] A. N. Baushev, Mon. Not. R. Astron. Soc. 417, L83 (2011).

[19] A. N. Baushev, arXiv:1205.4302.

[20] P. Gondolo, J. Edsjö, P. Ullio, L. Bergström, M. Schelke, and E. A. Baltz, J. Cosmol. Astropart. Phys. 07 (2004) 008.

[21] G. Jungman, M. Kamionkowski, and K. Griest, Phys. Rep. 267, 195 (1996).

[22] D.S. Gorbunov and V. A. Rubakov, Introduction to the Theory of the Early Universe: Hot Big-Bang Theory (World Scientific, Singapore, 2011).

[23] W. B. Atwood et al., Astrophys. J. 697, 1071 (2009).

[24] P. L. Nolan et al., Astrophys. J. Suppl. Ser. 199, 31 (2012), see also http://fermi.gsfc.nasa.gov/ssc/data/access/lat/2yr_ catalog/.

[25] Fermi Science Support Center, http://fermi.gsfc.nasa.gov/ ssc/data/analysis/software/aux/gal_2yearp7v6_v0.fits Fermi Science Support Center, http://fermi.gsfc.nasa.gov/ ssc/data/analysis/software/aux/iso_p7v6source.txt

[26] The Cherenkov Telescope Array, http://www .ctaobservatory.org/. 